\title{
Distributed Spectrum Access with Energy Constraint for Heterogeneous Channels
}

\author{
Miurel Tercero ${ }^{1,2}$, Pamela González Sánchez ${ }^{1,2}$, Ömer Ileri ${ }^{3}$, Jens Zander ${ }^{1}$ \\ ${ }^{1}$ The Royal Institute of Technology KTH, Radio Communication Systems, SE-164 40 Kista, Sweden \\ ${ }^{2}$ National University of Engineering (UNI), P.O. Box 5595, Managua, Nicaragua \\ ${ }^{3}$ The Scientific and Technological Research Council of Turkey (TÜBİTAK) \\ Emails: mitv@kth.se,dpgs@kth.se, omer.ileri@uekae.tubitak.gov.tr, jenz@kth.se
}

\begin{abstract}
The demand for wireless communications services has increased the amount of spectrum resources required, promoting research interest in dynamic spectrum allocation schemes. There exist many promising solutions to allocate spectrum on a dynamic basis in order to get an efficient spectrum usage. One particular form is auction mechanism, tailored for allocating transmission rights on a short term basis to provide efficient allocation of scarce resources. However, most existing approaches are focused on homogeneous settings where all channels are treated as if they have the same propagation characteristics. In this work we consider two distributed auction schemes; sequential and concurrent, based on sequential ascending and combinatorial bidding, respectively, taking into account the propagation conditions of the channels (heterogeneous settings). The performance of these schemes is compared to two reference cases: (i) distributed homogeneous channels allocation (channel-agnostic case), and (ii) the centralized allocation scheme. Experimental results suggest that auction mechanisms which take into account the channel characteristics improve spectrum utilization under energy constraint. For wireless cellular networks with high unit energy cost (low coverage) concurrent access gives a better utilization of the spectrum and energy resources with higher service providers utility.
\end{abstract}

\section{INTRODUCTION}

The rapid pace of technological development recently creates a need for more efficient use of available spectrum resources. The currently enforced spectrum management mechanisms mostly rely on static allocation of frequency bands across consumers (operators), in which a service provider (SP) gets usage rights for a specific band with long term dedication. Such static allocation mechanisms guarantee interference-free operation for the SP. Recent studies, on the other hand, suggest that static spectrum allocation methods are indeed inefficient in terms of spectrum utilization [1], especially when the demand drastically changes over the time. Then dynamic access mechanisms are needed in order to balance the demand with the supply.

Therefore, developing new schemes for dynamic spectrum access (DSA), aiming to avoid inefficiencies in the traditional licensing, has attracted significant interest recently. Fig.1 shows a schematic comparison for the trends of spectrum allocation mechanisms, characterized by two dimensions; performance and complexity. The complexity of a dynamic spectrum access system is likely to increase as the systems require more coordination, resulting in greater overhead due to control signalling. Such increased complexity, however, is likely to bring improvements in spectrum utilization efficiency.

Most of the earlier contributions on DSA schemes are concentrated on scenarios where the channels are considered as identical resources (channel agnostic), since all of them are assumed to have the same propagation characteristics, [2]-[5].
In such scenarios, DSA mechanisms are often implemented in the form of auctions where the prices for the different channels do not differ. Rather the auctioneer keeps increasing the unit cost for the channels until the total bandwidth demand is less than the total bandwidth supply.

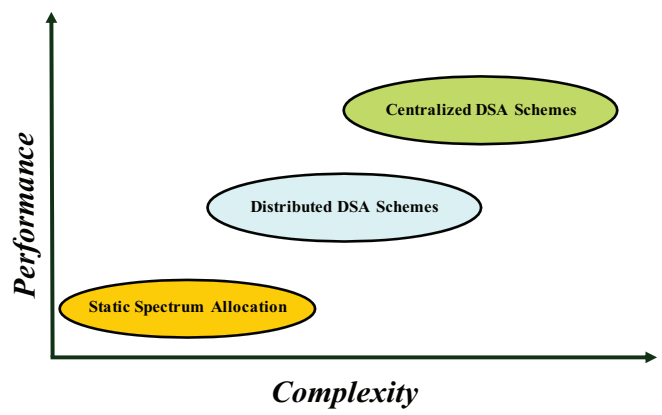

Fig. 1. Schematic comparison of different spectrum allocation mechanisms from two dimensions (performance,complexity)

Given the increased awareness about the excessive energy consumption of the communication systems, the "green radio" paradigm has recently started to emerge. It is likely that the energy consumption will become a major concern even for down-link transmissions in infrastructure systems [6]. Consequently, the propagation characteristics of available channels should be considered in making spectrum allocation decisions.

In this work, we consider two distributed DSA mechanisms that take into account the different propagation characteristics and power requirements of different channels; Sequential and Concurrent spectrum access. Similar schemes have also been investigated in [4], where the author has instead considered a channel-agnostic setting where the SPs determine their bids for the channels without any consideration of the propagation conditions.

The novelty of our work is to provide different means of propagation-aware DSA mechanisms (heterogenous channels) and show how they compare to each other with changing number of users for different energy cost levels. More specifically, we consider two regimes of interest, the first one is when the transmission energy cost (the monetary cost of each unit of energy required to transmit) is low, potentially resulting in a wireless network with large coverage area, and the second one when transmitting represents high cost corresponding to a wireless network which has poor coverage.

Only a few of the previous studies on DSA schemes [7][9] have considered heterogeneous channel settings, making the assumption that the channels have the same bandwidth 
but different propagation characteristics. Therefore, the users experience different transmission ranges, and thus different frequencies are suitable for different user locations. These studies focus on the description of how channel heterogeneity can be addressed on DSA. However, they do not evaluate the effect of energy cost on spectrum utilization efficiency.

We compare the performance of the considered two schemes to the centralized allocation scheme and the distributed homogeneous channels scheme which provide the upper and lower bound regarding to spectrum utilization, respectively.

The rest of the paper is organized as follows. In Section II we present the system model describing the basic assumptions and the operator revenue function used in our approaches. The auction mechanisms and the algorithms applied for the spectrum access schemes are described in detail in Section III. In Section IV we present the simulation results and finally, Section V summarizes the conclusion of this work.

\section{System MODEL}

\section{A. Basic assumptions}

We consider a cellular system scenario as illustrated in Fig.2, where users are randomly located with uniform distribution on a flat circular geographical region with cell radius $\mathrm{R}$. There are two service providers (SPs), each with one base station and their own predefined set of users $i=\{1,2, \ldots, I\}$, and a broker who represents the regulatory body responsible for spectrum allocation, with a set of available channels $j=\{1,2, \ldots, J\}$. The users want to get service and they can be served only by the operator that they belong to. The broker charges the operators for their channel usage, and it is responsible to gradually increase the channel prices (either in the form of sequential or combinatorial auctions), till there is no contention by the service providers for any of the channels.

The system is session based where a session is defined as the time duration for which the link gains in the system are constant or moderately changing. In our model we consider a simple distance based link gain model, therefore in the context of this study, a session is initiated each time there is a change in the location of any one of the users in the system. Note, however, that the concepts and mechanisms discussed in this paper are applicable in settings where link gains are timevarying, by considering the mean link gains over certain time intervals instead of taking the exact instantaneous link gain for a given user. The auction mechanisms are initiated at the beginning of each session and are finalized when all the channels are auctioned. The determined channel allocations are then valid for the rest of the session till a new session is initiated.

For the sake of simplicity, only downlink transmission is considered in this work. At the beginning of each session, the SPs consider the set of available channels and the corresponding link gains to their users over these channels. SPs are motivated to maximize their profit by choosing the most suitable user - channel combinations in the system, considering the cost for using the channel, as well the energy consumption that depends on the propagation characteristics of the channels. The auctions result in an interference-free system where each channel is occupied by at most one SP-user pair at any time instance.

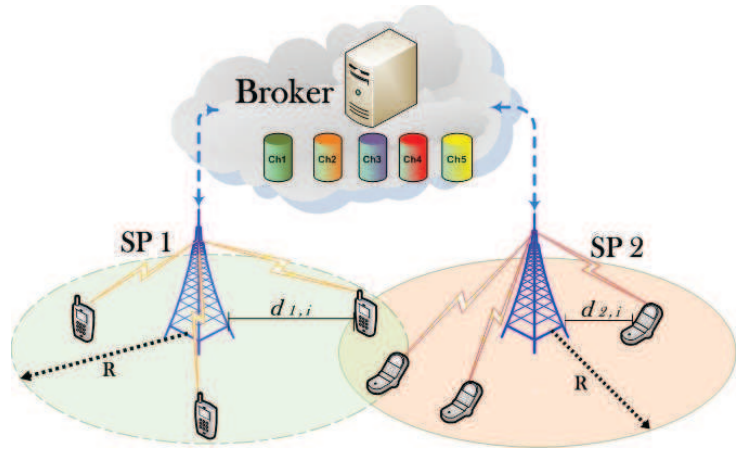

Fig. 2. Wireless Network Structure

\section{B. Channel Model}

The free space propagation model is used to deduce the required power transmission for a given service provider $\mathrm{r}$ $\left(S P_{r}\right)$ in order to reach the single user $i$ using the channel $j$ (frequency $f_{j}$ ), as shown in Eq.(1).

$$
P_{(r, i, j)}=\operatorname{Co.}\left(d_{r, i}\right)^{\alpha} \cdot\left(f_{j}\right)^{2}
$$

Where $C_{O}$ is a constant computed from the SNR threshold, noise power and the speed of light, $d_{r, i}$ is the distance between the base station of the $S P_{r}$ and the user $i$. Note, the dependence of $P_{(r, i, j)}$ on the propagation characteristic of channel $j$ that is being utilized and the user location.

\section{User Perspective}

The users have quality of service (QoS) requirement in the form of received signal to noise (SNR) threshold value. We also assume that each SP base stations employ power control so that the transmit power at a given channel is the minimum power sufficient for achieving the required SNR values at the designated end user.

\section{Service Provider Perspective (Revenue function)}

An important parameter in our algorithm that addresses the result from the auction process is the revenue (utility) function $U_{(r, i, j)}$. This represents the profit that the considered provider $S P_{r}$ achieves by serving user $i$ on channel $j$. In our model a revenue function is considered as shown in Eq.(2), which is used as a decision-taking element by the SPs, thus the service is provided to those users which promote positive utility $\left(U_{(r, i, j)}>0\right)$. The utility that a $S P_{r}$ perceives by serving the user $i$ in the channel $j$ is defined as follows:

$$
U_{(r, i, j)}=x_{u s e r}-C_{\operatorname{cost}(j)}-K \cdot P_{(r, i, j)}
$$

Where $x_{\text {user }}$ is a fixed price that each user pays to the SPs for the service, $C_{\operatorname{cost}(j)}$ is the channel cost that keeps varying during the auctioning phase (in monetary units) and $K$ is the energy cost per session in monetary unit/power unit . It can be viewed that high values of $K$ (expensive energy) relates to situations where SPs have limited coverage due to excessive expense of transmission, then the SPs are restricted to serve the nearer users. Meanwhile low values of $K$ (inexpensive energy) represents scenarios where SPs have relatively high coverage. 


\section{E. Performance Measures}

In order to evaluate the performance of the system the following parameters are considered:

1. Channel Occupancy[\%] is the total number of channels allocated to the SPs in the system as a result of the auctioning phase expressed in percentage. The channel occupancy is $100 \%$ when all the channels are assigned.

2. Normalized Power consumption per active user is the average power that each base station expends to serve a single user, achieving the SNR threshold. The expended power depends on the propagation characteristic of selected channels and the location of the users.

3. Service Provider Utility[\%] represents the SPs' average profit per each served user in the system.

\section{MECHANISM DESCRIPTION}

\section{A. Broker-SP Interaction}

The broker mediates the SP competition for the available channels. We consider two different auction mechanisms, sequential ascending for the distributed sequential access scheme and combinatorial iterative for the distributed concurrent access scheme. In order to solve the possible channel conflicts between the SPs the broker keeps setting (increasing or decreasing) the price for the channels for which there is contention until the conflicts are solved. The auction proceeds either in a simultaneous manner, as in distributed concurrent access scheme, or on a channel-by-channel basis, as in distributed sequential access scheme.

\section{B. Distributed Sequential and Concurrent Access schemes}

In this study we consider a two SPs setting. Note, however, that the mechanisms considered here are applicable to settings in which there are more SPs. As soon as the auction process is over, each SP is assigned a set of different channels, then the SPs start a session with their respective users. In the following we describe the two distributed schemes which are compared with the homogeneous and the centralized schemes.

Distributed Sequential Access scheme: In this scheme the spectrum access is based on sequential ascending auction. The broker offers the channels one by one on an individual basis. The competition is executed for one channel without any information about the likely price for the following channels.

Particularly in this model, the sequential access provides an inefficient channel allocation because the SPs can win the "wrong" channel at very high cost. Such phenomena is also mentioned in [10], which points out the fact that SPs are aware of this risk and they tend to respond by bidding more conservatively. Thus, the order in which the available channels are offered would make a difference. In this mechanism the channels are auctioned starting with the lowest frequency in ascending order in carrier frequency. We believe it is intuitive to start auctioning the channels with the greatest demand which are the channels with better propagation conditions (lower frequency) meaning that less power is required.

The auction is coordination-based where the broker is increasing the price $(\Delta 1)$, for a specific channel as long as both SPs are still interested in acquiring the channel. The process is executed till the price gets high enough so that only one of the SPs is still interested in the channel, and a consensus on spectrum allocation decision is reached, but there exist the risk that none of SPs get the channel.

The winner SP is charged with the prevailing price for the obtained channel and it can start a session transmission $\left(\mathrm{SP}_{-}\right.$ user) with its user. Note also that for any given channel, while making their bids the SPs select the remaining user which would require the minimum energy, in order to achieve the greatest possible revenue as expressed in Eq.(2).

In every iteration, each SP communicates the indicator variable $a_{r}$ to the broker, when $a_{r}=1 \mathrm{SPr}$ is still interested in the channel, and $a_{r}=0$ represents the lack of interest. The algorithm that performs this scheme is summarized as below.

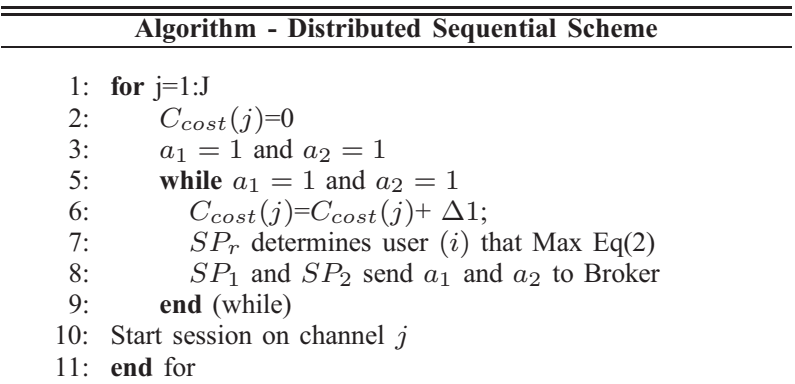

Distributed Concurrent Access scheme; The spectrum access is carried out by iterative combinatorial auction. The SPs are bidding for a package of different channels. The SPs compute the best user-channel combinations based on channels propagations characteristics, the location of their users and the channel costs as declared by the broker. In order to formulate the problem we define the combinatorial variable $a_{(r, i, j)} \in\{1,0\}$, such that $a_{(r, i, j)}=1$ if channel $j$ is assigned to user $i$ for $S P_{r}$ and $a_{(r, i, j)}=0$ otherwise, thus $S P_{r}$ finds the maximum revenue from the whole system by:

$$
\operatorname{Max} \sum_{i, j}^{I, J} a_{(r, i, j)} U_{(r, i, j)} .
$$

Two constraints that should be considered are that for any user $i$ a maximum of one channel should be assigned and any channel $j$ cannot be allocated to more than one user (as defined in $\operatorname{Eq}(4)$ and $\operatorname{Eq}(5)$, respectively).

$$
\begin{aligned}
& \sum_{r, j}^{R, J} a_{(r, i, j)} \leq 1 \\
& \sum_{i}^{I} a_{(r, i, j)} \leq 1
\end{aligned}
$$

The auction is implemented in multiple rounds where in each round each $S P_{r}$ computes its preferences and submits a channel request list $\overline{a_{(r, j)}}$ to the Broker. This channel list does not include user index since this is a concern for SPs only. At the end of each iteration, the broker determines the channels for which there is contention $\left(\overline{a_{(1, j)}} \cap \overline{a_{(2, j)}}\right)$, increases the price for them at $\Delta 1$ while the prices for the channels that are not demanded are decreased by $\Delta 2$ to make 
them attractive for the SPs. The algorithm for Distributed Concurrent Access scheme is described as below.

\begin{tabular}{ll}
\hline \hline & Algorithm - Distributed Concurrent Scheme \\
\hline 1: & $C_{\text {cost }}=0$ \\
2: & Initially $\overline{a_{(1, j)}} \cap \overline{a_{(2, j)}} \neq$ null: Ch-Conflict \\
3: & while1 Ch-Conflict \\
4: & $C_{\text {cost }}($ Ch-Conflict $)=C_{\text {cost }}($ Ch-Conflict $)+\Delta 1 ;$ \\
5: & $C_{\text {cost }}($ Ch-Free $)=C_{\text {cost }}($ Ch-Free $)-\Delta 2 ;$ \\
6: & Each $S P_{r}$ determine $(i, j)$ pairs that Max Eq(3) \\
7: & $S P_{1}$ and $S P_{2}$ send $\overline{a_{(1, j)}}$ and $\overline{a_{(2, j)}}$ to Broker \\
8: & end (while1) \\
9: & Start session on all channels
\end{tabular}

\section{Reference Mechanisms}

Distributed Homogeneous Access Scheme: It is an auction-based homogeneous channels allocation mechanism that is modeled as a special case of the concurrent scheme with the variation that all channels are priced for the same amount meaning that the price is increased for all the channels at $\Delta 1$. In this sense the set of channels are auctioned as homogeneous resources even though the energy consumption in all the channels is different. Note that such an algorithm is representative of schemes where the auctioneer keeps increasing prices for all the channels as long as there is contention in any one of the available channels. The algorithm for Distributed Homogeneous Access scheme is described as follows.

\begin{tabular}{ll}
\hline \hline & Algorithm - Distributed Homogeneous Scheme \\
\hline 1: & $C_{\text {cost }}=0$ \\
2: & Initially $\overline{a_{(1, j)}} \cap \overline{a_{(2, j)}} \neq$ null: Ch-Conflict \\
3: & while1 Ch-Conflict \\
4: & $C_{\text {cost }}($ All-Channels $)=C_{\text {cost }}$ (All-Channels) $+\Delta 1 ;$ \\
5: & Each $S P_{r}$ determine $(i, j)$ pairs that Max Eq(3) \\
6: & $S P_{1}$ and $S P_{2}$ send $\overline{a_{(1, j)}}$ and $\overline{a_{(2, j)}}$ to Broker \\
7: & end (while1) \\
8: & Start session on all channels
\end{tabular}

Centralized Access Scheme; The decision-making process is led by the spectrum broker who is responsible for allocating spectrum resources among the SPs. It is a full knowledge system which gives the upper bound in spectrum utilization. The broker determines the optimum SP-userchannel associations that maximizes the total income of the SPs meaning that the total energy expenditure in the system is the minimum value. Moreover, here the channel cost is zero and the allocation process is implemented with no SP competition. In this scheme the SPs communicate the link gain vector for their end users, $\overline{g_{i}^{r}}$ to the broker. A basic algorithm has been developed and is described as follows:

\begin{tabular}{l}
\hline \hline \multicolumn{1}{c}{ Algorithm - Centralized Scheme } \\
\hline 1: $S P_{1}$ and $S P_{2}$ send $\overline{g_{i}^{r}}$ to Broker \\
2: $\begin{array}{l}\text { Broker determines the }\left(S P_{r}, i, j\right) \text { triplets for both op- } \\
\text { erators, such that total revenue of both operators are } \\
\text { maximized }\end{array}$ \\
3: Start session on all channels
\end{tabular}

It is relevant to mention that this is a hypothetic dynamic scheme, given that the allocation is not static. However, this scheme has the shortcoming regarding truthful information reporting by the SPs to the Broker. It is possible that the SP reacts selfishly and reports a suitable link gain vector in order to get the resources of interest. Moreover the system requires a high complexity design, high setup delays, and suffers single point of failure and non scalability. In this situation the distributed schemes are better options.

\section{NumericAl Results}

\section{A. Simulation Settings}

In this section we present the numerical experiments that we conducted in a two SPs setting where each SP has a maximum of seven users. The system has five available channels with carrier frequencies as indicated in Table I, which summarizes the simulation parameters.

TABLE I

Simulation Parameter Value

\begin{tabular}{|l|l|}
\hline PARAMETERS & VALUES \\
\hline Propagation exponent, $\alpha$ & 2 \\
\hline Users distribution & Uniform \\
\hline Energy Cost, K [monetary unit/power unit] & {$[10,1000]$} \\
\hline User Payment, $P_{\text {user }}[$ monetary unit] & 5 \\
\hline Channel Price, $C_{\operatorname{cost}(j)}[$ monetary unit] & {$[0-5]$} \\
\hline Number of Channels & 5 \\
\hline Number of SPs & 2 \\
\hline Carrier Frequencies $[\mathrm{GHz}]$ & $0.6,1.3,1.7$ \\
& $1.9,2.5$ \\
\hline
\end{tabular}

\section{B. Simulation Results}

1. Channel Occupancy; From Fig. 3 the main findings can be summarized as follows: The results from concurrent scheme show that by auctioning the channels based on their propagation conditions (discriminatory pricing is applied) a considerable gain is provided in terms of spectrum occupancy. When low value of energy cost $(K=10)$ is considered sequential and concurrent mechanisms present almost the same performance as the Centralized in terms of channel occupancy, allocating the maximum possible number of channels. By assuming that the value of energy cost increases $(\mathrm{K}=1000)$ a greater difference between sequential and concurrent schemes can be observed, showing that the concurrent access almost approaches the upper bound (the centralized allocation).

2. Normalized Power Consumption per active user; Fig. 4 shows that the power consumption in the homogeneous scheme is the lowest in the system since the channel occupancy is the minimum compared to other schemes. The power consumption that the sequential access mechanism expends per each served user is the highest since all the channels are auctioned one by one consecutively and SPs do not know whether or not the next channel is better.

In general the power expenditure tends to decrease as a function of the distance between the base station and the served users, $d_{r, i}$. As there are more users in the system, the probability that the served users get nearer to the base station increases and so the power consumption gets lower. 


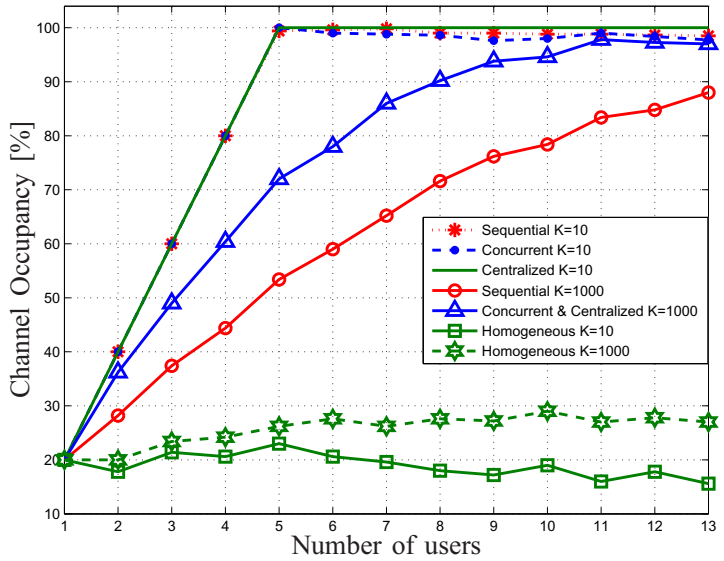

Fig. 3. Channel Occupancy in the System versus number of users

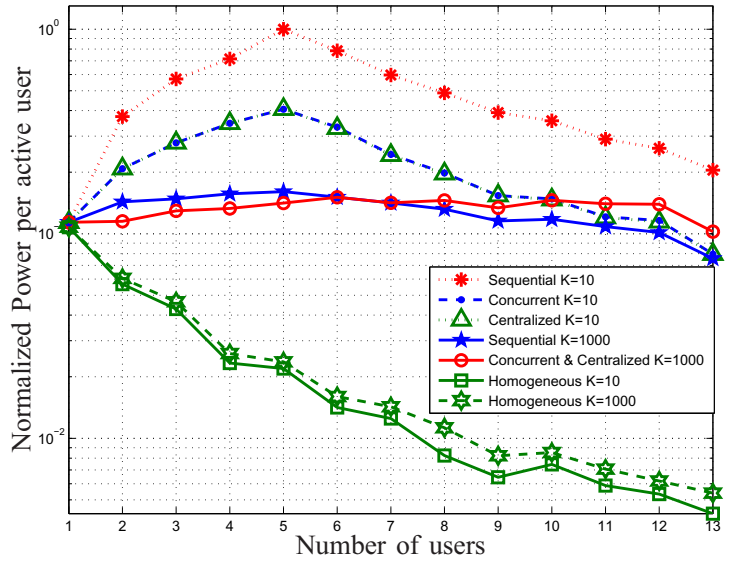

Fig. 4. Power expended per each served user in the System

3. Service Provider Utility; The maximum utility that a SP can perceive by serving a user is obtained from the Centralized mechanism as it was expected since the channel price is considered zero, see Fig.5. However, when the channel price is taken into account in the distributed mechanisms, the concurrent access gives higher utility.

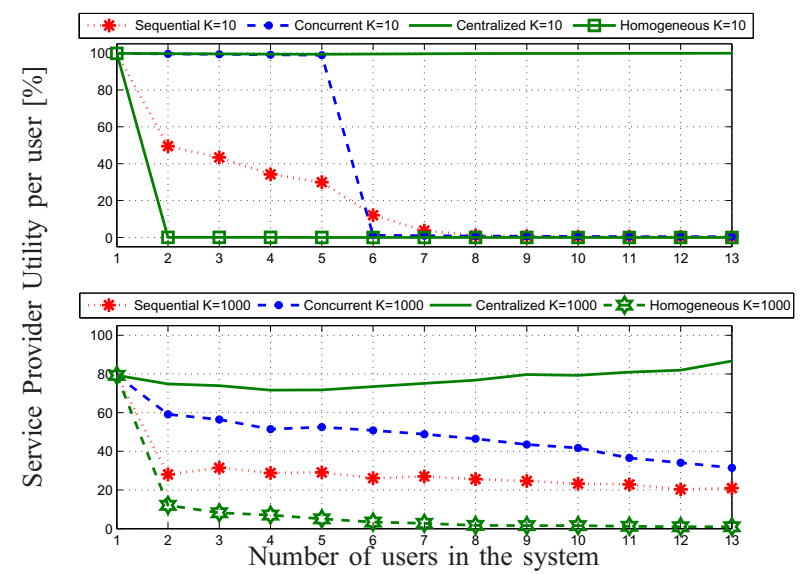

Fig. 5. Service provider utility per served user in the System
Furthermore, for low energy cost $(\mathrm{K}=10)$ in the concurrent and sequential access the channel cost, $C_{\text {cost }}$, increments as a function of the number of users in overload situation. Subsequent to this fact the SPs utility starts to decrement radically after five users. From this graph we may conclude that the strategy used in distributed sequential scheme is not that efficient, and some other cooperation strategy may lead to better profits.

\section{CONCLUSION}

We considered two distributed DSA schemes, based on auction methods with energy constraint for heterogeneous channels settings. The channel heterogeneity proves to be an important fact to take into account in order to improve the spectrum occupancy. Based on the energy constraint the SPs have to be aware of the power expenditure when choosing the userchannel pairs. In the case of low unit energy cost K (good coverage) the performance of the sequential and concurrent access schemes approaches the upper bound (centralized access) regarding to spectrum utilization. Furthermore, when the unit energy cost is high (limited in coverage) the sequential access gives higher power consumption and lower SPs' utility due to inefficient channels allocation. Hence, we summarize that when it comes to the case of wireless network with energy constraint the concurrent access scheme is the better option for DSA.

\section{ACKNOWLEDGMENT}

The authors would like to acknowledge the Swedish International Development Agency (Sida), who has supported in part this work.

\section{REFERENCES}

[1] M. Pattal and J. Zeng, "Spectrum management reforms: A step towards a flexible spectrum management," in Wireless Communications, Networking and Mobile Computing, WiCOM 08, 4th International Conference on, 2008.

[2] O. Ileri, D. Samardzija, and N. Mandayam, "Demand responsive pricing and competitive spectrum allocation via a spectrum server," in New Frontiers in Dynamic Spectrum Access Networks, 2005. DySPAN 2005. 2005 First IEEE International Symposium on, Nov. 2005.

[3] S. Sengupta, M. Chatterjee, and S. Ganguly, "An economic framework for spectrum allocation and service pricing with competitive wireless service providers," in New Frontiers in Dynamic Spectrum Access Networks, 2007. DySPAN 2007. 2nd IEEE International Symposium on, 2007.

[4] S. Sengupta and M. Chatterjee, "Sequential and concurrent auction mechanisms for dynamic spectrum access," in Cognitive Radio Oriented Wireless Networks and Communications, 2007. CrownCom 2007. 2nd International Conference on, Aug. 2007, pp. 448-455.

[5] G. Sorabh, B. Chiranjeeb, C. Lili, Z. Haitao, and S. Subhash, "Toward real-time dynamic spectrum auctions," Computer Networks, vol. 52, pp. 879-897, 2008

[6] Kungliga Tekniska Hgskolan, "Research project: Green radioinvestigating energy consumption of wireless networks," Available: http://www.wireless.kth.se/projects/GREEN/index.php, 2009.

[7] M. Ma and D. H. K. Tsang, "Impact of channel heterogeneity on spectrum sharing in cognitive radio networks," in Communications, 2008. ICC '08. IEEE International Conference on, 2008.

[8] J. Sun and E. Modiano, "Wireless channel allocation using an auction algorithm," (IEEE) Journal on selected areas in Communication, vol. 24, no. 5,2006

[9] V. Brik, E. Rozner, and S. Banerjee, "Dsap: a protocol for coordinated spectrum access," in IEEE DySPAN, 2005, pp. 611-614.

[10] C. Kolboldt, D. Maldoom, and R. Marsden, "The first combinatorial spectrum auction, lessons from the nigerian auction of wireless access licences," in London W1S - $1061 D N, 2003$. 